\title{
Hábitos y Trastornos Alimenticios asociados a factores Socio- demográficos, Físicos y Conductuales en Universitarios de Cartagena, Colombia
}

\author{
Shirly Sáenz Durana, Farith González Martínez ${ }^{b}$, Shyrley Díaz Cárdenas ${ }^{c}$
}

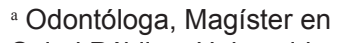
Salud Pública, Universidad Nacional de Colombia.

b Odontólogo, Magíster en Salud Pública, Departamento de Investigación, Facultad de Odontología, Universidad de Cartagena, Colombia.

' Odontóloga, Magíster en Salud Pública, Departamento de Odontología Preventiva y social, Facultad de Odontología,

Universidad de Cartagena, Colombia.

\section{Correspondencia:}

Farith González Martínez,

Facultad de Odontología,

Universidad de Cartagena,

Campus de la Salud, barrio

Zaragocilla, Cartagena

de Indias, Colombia. Telf.:

6698184 ext. 110, correo

electrónico: farithgm@hotmail. com.

Recibido el 9 de julio de 2011.

Aceptado para su publicación el 1 de agosto de 2011

\section{RESUMEN}

Objetivo. Describir la prevalencia de hábitos y desórdenes alimenticios en universitarios y relacionarlos con factores socio-demográficos, físicos y conductuales.

Diseño. Estudio de corte transversal.

Emplazamiento. Municipio de Cartagena de Indias, Colombia.

Participantes. Un total de 1.040 estudiantes de los programas diurnos y nocturnos de la Universidad de Cartagena.

Mediciones principales. Se utilizó una encuesta estructurada para evaluar hábitos alimenticios y aspectos socio-demográficos, EAT-26 y SCOFF para trastornos alimenticios y escala de Holmes and Rahe para la susceptibilidad de enfermar. Se estimó la ocurrencia a través de prevalencias, las relaciones entre variables a través de razones de disparidad y el análisis multivariable mediante regresión logística nominal.

Resultados. El motivo más frecuente para no alimentarse bien fue la falta de costumbre y tiempo. La susceptibilidad de enfermar fue del $23,1 \%$ y el riesgo de anorexia y bulimia del $14,2 \%$. En el análisis multivariable el mejor modelo con el SCOFF mostró significación estadística con edad (OR: 1,86), susceptibilidad de enfermar (OR: 1,77), dieta (OR: 2,81$)$, problemas de colon (OR: 1,8$)$ y no realizar actividad física (OR: 3,04 ).

Conclusiones. Los hábitos y trastornos de alimentación en estudiantes universitarios están influidos por factores relacionados con el quehacer universitario, siendo necesario considerar este comportamiento alimentario como un problema relevante que interactúa con el buen desarrollo de las actividades académicas.

Palabras Clave. Hábitos Alimenticios, Trastornos Alimenticios, Estudiantes.

\section{ABSTRACT}

Eating habits and disorders associated to socio-demographic, physical and behavioural factors in university students, Cartagena Colombia.

Objective. To describe the prevalence of eating habits and disorders in university students and their relationship with socio-demographic, physical and behavioural factors.

Design. Cross-sectional study

Setting. Municipality of Cartagena de Indias, Colombia.

Participants. 1040 students in day and evening programmes at the University of Cartagena.

Main measurements. A structured questionnaire was used to assess eating habits and sociodemographic factors; EAT-26 and SCOFF for eating disorders and the Holmes and Rahe scale for the susceptibility to disease. Occurrence was estimated by prevalence, assuming confidence $95 \%$ intervals. Relationships between variables were evaluated using odds ratios and multivariate analysis using nominal logistic regression.

Results. The most common reason for not eating well was the lack of habit and time. Susceptibility to disease was $23.1 \%$ and the risk of anorexia and bulimia $14.2 \%$. The best model for logistic regression with SCOFF showed statistical significance with age (OR: 1.86), susceptibility to disease (OR: 1.77), diet (OR: 2.81), colon problems (OR: 1.8), and lack of physical activity (OR: 3.04).

Conclusions. Eating habits and disorders in university students are influenced by factors related to university life. This behavior should be considered as a serious problem that one that interferes with the smooth running of academic activities.

Key Words. Eating Habits, Eating Disorders, Students. 


\section{INTRODUCCIÓN}

El estilo de vida de determinados grupos poblacionales puede conducir a hábitos alimentarios y modelos dietéticos y de actividad física que se convierten en factores de riesgo para las enfermedades crónicas. Por otro lado, el acceso a la universidad supone un cambio importante en el individuo, que puede repercutir en su estilo de vida, lo que convierte a este subgrupo poblacional en vulnerable desde el punto de vista nutricional ${ }^{1}$. Los trastornos del comportamiento alimentario son problemas graves que producen complicaciones médicas y psicosociales que pueden llevar a la muerte. Se cree que en los países occidentales industrializados la frecuencia de los trastornos de la conducta alimentaria ha aumentado en las últimas tres décadas y que la edad de inicio es más temprana. Los trastornos de la conducta alimentaria entre mujeres adolescentes y adultos jóvenes de estos países presentan una prevalencia que oscila entre el $1 \%$ y el $8 \%$, siendo más frecuente la bulimia nerviosa que la anorexia nerviosa ${ }^{2,3,4}$.

Los estudios realizados en Colombia sobre hábitos alimenticios en estudiantes universitarios son escasos y la mayor parte de éstos utilizan instrumentos de cribado para estudiar la prevalencia de riesgo de la enfermedad. En otros países se han reportado estudios por García-Segovia ${ }^{5}$, cuyos resultados demuestran que la comida del mediodía es percibida como la más importante y un alto porcentaje de los encuestados la consumen fuera de su lugar de residencia, al menos dos veces por semana. En este mismo sentido, la actividad física diaria de los participantes fue considerada como media, siendo los hombres los que más deporte realizan. Estas exposiciones son consideradas un problema de salud pública en Colombia, debido a que el sobrepeso y la obesidad tienen aún mayor relevancia. Son potencialmente eventos silenciosos que tienen consecuencias catastróficas en la salud general de una población, siendo más preocupante en el entorno de la región Caribe Colombiana, cuya población, por influencias comportamentales mediadas por la cultura, ha trasformado sus hábitos alimenticios con dietas similares a las de países en desarrollo y la mayoría de sus adolescentes llevan un estilo de vida sedentario . $^{\text {. }}$

En la ciudad de Cartagena no se conocen publicaciones que aborden esta problemática, aunque se han reportado estudios en tópicos muy generales sobre el comportamiento de los estudiantes, relacionados con su sexualidad y consumo del cigarrillo entre otros, pero no se ha indagado a fondo sobre los hábitos y desordenes alimenticios de los mismos, sus causas y posibles impactos en la salud.

Teniendo en cuenta estos hallazgos, interferir los estilos de vida se convierte en una de las acciones más eficaces para la prevención de enfermedades de tipo metabólico y cardiovascular y la promoción de la salud general de un individuo, siendo importante promover en los estudiantes universitarios los estilos de vida saludables, los cuales se ven influidos por variables fisiológicas, psicosociales y familiares.

El objetivo del presente estudio fue describir la prevalencia de hábitos y desórdenes alimenticios en universitarios y relacionarlos con factores sociodemográficos, físicos y conductuales.

\section{MATERIAL Y MÉTODO}

Estudio de corte transversal realizado en una población de 8.981 estudiantes matriculados en la universidad de Cartagena, sede Cartagena de Indias, en jornadas diurnas y nocturnas presenciales en el primer periodo académico de 2011. La muestra fue de 980 sujetos, cálculo obtenido a partir de una fórmula estadística, asumiendo una frecuencia esperada del fenómeno de estudio (trastornos alimenticios) del 15\%, confianza del $95 \%$ y error relativo del $2 \%$. Este tamaño se recalculó teniendo en cuenta una tasa de no respuesta del $5 \%$, obteniéndose una muestra definitiva de 1.040 participantes.

El muestreo utilizado fue de tipo probabilístico con afijación proporcional por programas y semestre con reemplazo. Para tal fin se utilizó una lista de números aleatorios con la cual se fueron seleccionando los individuos. Previo a esto se tuvo en cuenta que los participantes fueran estudiantes activos matriculados en la universidad de Cartagena durante el periodo de estudio. Se excluyó a los estudiantes que no desearon participar en el estudio, a los que no diligenciaron el consentimiento informado por escrito y a los que no emitieron de forma adecuada las respuestas en el cuestionario.

Se utilizó una encuesta estructurada para valorar los hábitos alimenticios y las variables sociodemográficas (variables independientes), la cual consta de 32 preguntas politómicas tipo Likert con 


\begin{tabular}{|c|c|c|}
\hline Variables & Número ( $n=1.040)$ & Porcentaje (\%) \\
\hline \multicolumn{3}{|l|}{ Grupo de Edad } \\
\hline - $\quad<19$ años (adolescente) & 493 & 47,4 \\
\hline - $20-44$ (adulto joven) & 547 & 52,6 \\
\hline \multicolumn{3}{|l|}{ Sexo } \\
\hline - Femenino & 527 & 50,7 \\
\hline - Masculino & 513 & 49,3 \\
\hline \multicolumn{3}{|l|}{ Semestre } \\
\hline - Primero & 138 & 13,3 \\
\hline - Segundo & 100 & 9,6 \\
\hline - Tercero & 87 & 8,4 \\
\hline - Cuarto & 192 & 18,5 \\
\hline - Quinto & 188 & 18,1 \\
\hline - Sexto & 91 & 8,7 \\
\hline - Séptimo & 88 & 8,5 \\
\hline - Octavo & 80 & 7,7 \\
\hline - Noveno & 44 & 4,2 \\
\hline - Décimo & 32 & 3,1 \\
\hline \multicolumn{3}{|l|}{ Programas } \\
\hline - Odontología & 78 & 7,5 \\
\hline - Economía & 49 & 4,71 \\
\hline - Enfermería & 49 & 4,71 \\
\hline - Biología & 14 & 1,35 \\
\hline - Administración empresas & 94 & 9,04 \\
\hline - Administración industrial & 52 & 5,01 \\
\hline - Ingeniería Química & 38 & 3,65 \\
\hline - Contaduría & 100 & 9,62 \\
\hline - Ingeniería Civil & 54 & 5,19 \\
\hline - Historia & 49 & 4,71 \\
\hline - Ingeniería de Alimentos & 39 & 3,75 \\
\hline - Trabajo social & 27 & 2,6 \\
\hline - Química pura y farmacia & 73 & 7,02 \\
\hline - Matemáticas & 27 & 2,6 \\
\hline - Ingeniería de Sistemas & 29 & 2,79 \\
\hline - Derecho diurno & 98 & 9,42 \\
\hline - Comunicación social & 48 & 4,6 \\
\hline - Lingüística y literatura & 46 & 4,4 \\
\hline - Filosofía & 49 & 4,71 \\
\hline - Medicina & 27 & 2,63 \\
\hline \multicolumn{3}{|l|}{ Procedencia } \\
\hline - Urbana & 801 & 77 \\
\hline - Rural & 239 & 23 \\
\hline \multicolumn{3}{|l|}{ Convivencia } \\
\hline - Con personas diferentes & 171 & 16,4 \\
\hline - Con familiares & 827 & 79,5 \\
\hline - Solo & 42 & 4,1 \\
\hline \multicolumn{3}{|l|}{ Ingresos Económicos } \\
\hline - Menos de 1 SMMLV & 305 & 29,3 \\
\hline - Entre 1 y 3 SMMLV & 506 & 48,6 \\
\hline - Entre 4 y 5 SMMLV & 64 & 6,2 \\
\hline - Más de 6 SMMLV & 25 & 2,4 \\
\hline - Ningún ingreso & 140 & 13,5 \\
\hline
\end{tabular}

Tabla 1. Características de la muestra estudiada (Cartagena, 2011).

única respuesta. Ésta fue diseñada desde la teoría y fue evaluada su validez de apariencia a través de tres jueces expertos, teniendo en cuenta su pertinencia, suficiencia de las categorías, plausibilidad, semántica, sintaxis y ordenamiento de los ítems. Las variables sociodemogràficas fueron: edad en años cumplidos, sexo, estratificación social (bajo 1 y 2 , medio 3 y 4 , alto 5 y 6 ), programas académicos (derecho diurno, derecho vespertino, comunicación social, trabajo social, lingüística y literatura, historia, filosofía, economía, ingeniería civil, ingeniería de sistemas, ingeniería de alimento, administración de empresas, administración industrial, contaduría pública, matemáticas, medicina, odontología, enfermería, química farmacéutica y química) y semestres (I a X). 


\begin{tabular}{|c|c|c|c|}
\hline Hábitos alimenticios & Frecuencia $(n=1.040)$ & Porcentaje (\%) & IC $95 \%$ \\
\hline \multicolumn{4}{|l|}{ Tres comidas diarias } \\
\hline Siempre & 467 & 44,9 & $41,9-47,9$ \\
\hline Casi siempre & 374 & 36 & $33,0-38,9$ \\
\hline Algunas veces & 159 & 15,3 & $13,1-17,5$ \\
\hline Casi nunca & 34 & 3,3 & $2,2-4,4$ \\
\hline Nunca & 6 & 0,6 & $0,1-1,0$ \\
\hline \multicolumn{4}{|l|}{ Hábito del desayuno } \\
\hline Siempre & 509 & 48,9 & $45,9-60,0$ \\
\hline Casi siempre & 246 & 23,7 & $21,1-26,2$ \\
\hline Algunas Veces & 194 & 18,7 & $16,3-21,0$ \\
\hline Casi Nunca & 70 & 6,7 & $5,2-8,3$ \\
\hline Nunca & 21 & 2 & $1,2-2,9$ \\
\hline \multicolumn{4}{|c|}{ Hora de las comidas adecuadas } \\
\hline Siempre & 85 & 8,2 & $6,5-9,8$ \\
\hline Casi siempre & 371 & 35,7 & $32,8-38,6$ \\
\hline Algunas Veces & 371 & 35,7 & $32,8-38,6$ \\
\hline Casi Nunca & 156 & 15 & $12,8-17,2$ \\
\hline Nunca & 57 & 5,5 & $4,1-6,9$ \\
\hline \multicolumn{4}{|l|}{ Hora de desayuno } \\
\hline Entre las 5 y 6 am & 130 & 12,5 & $10,5-14,5$ \\
\hline Entre las 6 y 7 am & 332 & 31,9 & $29,1-34,8$ \\
\hline Entre las 7 y 8 am & 151 & 14,5 & $12,4-16,7$ \\
\hline Entre las 8 y 9 am & 352 & 33,9 & $31,0-36,7$ \\
\hline No desayuno & 75 & 7,2 & $5,6-8,8$ \\
\hline \multicolumn{4}{|l|}{ Lugar del almuerzo } \\
\hline Lugar de residencia & 633 & 60,9 & $57,9-63,8$ \\
\hline Universidad & 252 & 24,3 & $21,6-26,8$ \\
\hline En restaurantes & 133 & 12,8 & $10,8-14,2$ \\
\hline No almuerza & 22 & 2,2 & $1,2-3,0$ \\
\hline \multicolumn{4}{|c|}{ Lleva almuerzo de su residencia } \\
\hline Siempre & 32 & 3,1 & $2,0-4,1$ \\
\hline Casi siempre & 65 & 6,3 & $4,8-7,7$ \\
\hline Algunas Veces & 147 & 14,1 & $12,0-16,3$ \\
\hline Casi Nunca & 96 & 9,2 & $7,5-11,0$ \\
\hline Nunca & 700 & 67,3 & $6,5-7,2$ \\
\hline \multicolumn{4}{|c|}{ Asiste al comedor universitario } \\
\hline Siempre & 47 & 4,5 & $3,3-5,8$ \\
\hline Casi siempre & 98 & 9,4 & $7,6-11,2$ \\
\hline Algunas veces & 139 & 13,4 & $11,3-15,4$ \\
\hline Casi nunca & 92 & 8,9 & $7,1-10,6$ \\
\hline Nunca & 664 & 63,9 & $60,9-66,8$ \\
\hline \multicolumn{4}{|l|}{ Consumo de merienda } \\
\hline Si consumen & 605 & 58,2 & $55,2-61,2$ \\
\hline No consumen & 435 & 41,8 & $38,8-44,8$ \\
\hline
\end{tabular}

Tabla 2. Hábitos alimenticios diarios en los sujetos de estudio (Cartagena, 2011).

Para los trastornos alimenticios (variable dependiente) se utilizó el instrumento EAT-26 ${ }^{7}$, como prueba de despistaje, el cual consta de 26 preguntas no estructuradas, y posteriormente el cuestionario $\mathrm{SCOFF}^{8}$, el cual tiene cinco preguntas no estructuradas y evalúa los trastornos anorexia y bulimia. Para la susceptibilidad de enfermar (variable independiente) se utilizó la escala de Holmes And Rahe ${ }^{9}$, que evalúa 42 eventos que generarían alteraciones entre los jóvenes y con cuya puntuación se obtienen las siguientes probabilidades de enfermar: mayor de 300 (probabilidad de enfermar del $80 \%$ en un futuro cercano, 3 meses), $150-299$ (50\% de probabilidad de enfermar) y menor de 149 (30\% de probabilidad de enfermar).

La información fue recolectada en una sola etapa dentro de los programas y semestres muestreados. Los investigadores entregaron los cuestionarios a cada participante previo diligenciamiento del formato de consentimiento informado por escrito, el cual estuvo contemplado en el presente estudio a partir del trabajo con seres humanos y la posible violación de su intimidad con la divulgación de la 


\begin{tabular}{|c|c|c|c|}
\hline Tipo de alimento & Frecuencia $(n=1.040)$ & Porcentaje (\%) & IC $95 \%$ \\
\hline \multicolumn{4}{|c|}{ Cereales al desayuno } \\
\hline Siempre & 257 & 24,7 & $22,1-27,3$ \\
\hline Casi siempre & 252 & 33,8 & $31,0-36,7$ \\
\hline Algunas Veces & 109 & 10,5 & $8,6-12,3$ \\
\hline Casi Nunca & 236 & 22,7 & $20,1-25,2$ \\
\hline Nunca & 86 & 8,3 & $6,6-9,9$ \\
\hline \multicolumn{4}{|l|}{ Frutas al desayuno } \\
\hline Siempre & 142 & 13,7 & $11,6-15,7$ \\
\hline Casi siempre & 188 & 18,1 & $15,7-20,4$ \\
\hline Algunas Veces & 130 & 12,5 & $10,5-14,5$ \\
\hline Casi Nunca & 334 & 32,1 & $29,3-35,0$ \\
\hline Nunca & 246 & 23,7 & $21,1-26,2$ \\
\hline \multicolumn{4}{|c|}{ Carnes al desayuno } \\
\hline Siempre & 168 & 16,2 & $13,9-18,4$ \\
\hline Casi siempre & 175 & 16,8 & $14,5-19,1$ \\
\hline Algunas Veces & 103 & 9,9 & $8,1-11,7$ \\
\hline Casi Nunca & 242 & 23,3 & $20,7-25,8$ \\
\hline Nunca & 352 & 33,8 & $31,0-36,7$ \\
\hline \multicolumn{4}{|c|}{ Azucares al desayuno } \\
\hline Siempre & 286 & 27,5 & $24,8-30,2$ \\
\hline Casi siempre & 165 & 15,9 & $13,6-18,1$ \\
\hline Algunas Veces & 133 & 12,8 & $10,8-14,8$ \\
\hline Casi Nunca & 203 & 19,5 & $17,1-21,9$ \\
\hline Nunca & 253 & 24,3 & $21,7-26,9$ \\
\hline \multicolumn{4}{|c|}{ Cereales al almuerzo } \\
\hline Siempre & 248 & 23,8 & $21,3-26,4$ \\
\hline Casi siempre & 273 & 26,3 & $23,6-28,9$ \\
\hline Algunas Veces & 129 & 12,4 & $10,4-14,4$ \\
\hline Casi Nunca & 194 & 18,7 & $15,6-20,3$ \\
\hline Nunca & 196 & 18,8 & $12,1-16,4$ \\
\hline \multicolumn{4}{|l|}{ Frutas al almuerzo } \\
\hline Siempre & 154 & 14,8 & $12,6-17,0$ \\
\hline Casi siempre & 199 & 19,1 & $16,7-21,5$ \\
\hline Algunas Veces & 106 & 10,2 & $8,4-12,0$ \\
\hline Casi Nunca & 283 & 27,2 & $24,5-29,9$ \\
\hline Nunca & 298 & 28,7 & $25,9-31,4$ \\
\hline \multicolumn{4}{|l|}{ Carnes al almuerzo } \\
\hline Siempre & 471 & 45,3 & $42,3-48,3$ \\
\hline Casi siempre & 326 & 31,4 & $28,4-34,1$ \\
\hline Algunas Veces & 72 & 6,9 & $5,4-8,5$ \\
\hline Casi Nunca & 75 & 7,2 & $5,6-8,8$ \\
\hline Nunca & 96 & 9,2 & $7,5-11,0$ \\
\hline \multicolumn{4}{|c|}{ Vegetales al almuerzo } \\
\hline Siempre & 288 & 27,7 & $25,0-30,4$ \\
\hline Casi siempre & 291 & 28 & $25,2-30,7$ \\
\hline Algunas Veces & 126 & 12,1 & $10,1-14,1$ \\
\hline Casi Nunca & 187 & 18 & $15,6-20,3$ \\
\hline Nunca & 148 & 14,2 & $12,1-16,4$ \\
\hline \multicolumn{4}{|l|}{ Frutas en la cena } \\
\hline Siempre & 143 & 13,8 & $11,7-15,8$ \\
\hline Casi siempre & 175 & 16,8 & $14,5-19,1$ \\
\hline Algunas Veces & 121 & 11,6 & $9,7-13,6$ \\
\hline Casi Nunca & 338 & 32,5 & $29,6-35,4$ \\
\hline Nunca & 263 & 25,3 & $22,6-27,9$ \\
\hline \multicolumn{4}{|c|}{ Vegetales en la cena } \\
\hline Siempre & 125 & 12 & $10,0-14,0$ \\
\hline Casi siempre & 259 & 24,9 & $22,3-27,5$ \\
\hline Algunas Veces & 164 & 15,8 & $13,6-18,0$ \\
\hline Casi Nunca & 258 & 24,8 & $22,2-27,4$ \\
\hline Nunca & 234 & 22,5 & $20,0-25,0$ \\
\hline \multicolumn{4}{|l|}{ Carnes en la cena } \\
\hline Siempre & 314 & 30,2 & $27,4-33,0$ \\
\hline Casi siempre & 362 & 34,8 & $31,9-37,7$ \\
\hline Algunas Veces & 97 & 9,3 & $7,6-11,1$ \\
\hline Casi Nunca & 140 & 13,5 & $11,4-15,5$ \\
\hline Nunca & 127 & 12,2 & $10,2-14,2$ \\
\hline
\end{tabular}

Tabla 3. Tipo de alimentos consumidos durante el día en los sujetos de estudio (Cartagena, 2011). 


\begin{tabular}{|c|c|c|c|}
\hline Factores relacionados & Frecuencia $(n=1.040)$ & Porcentaje (\%) & IC $95 \%$ \\
\hline \multicolumn{4}{|l|}{ Motivo de sus hábitos alimenticios } \\
\hline Dificultades económicas & 132 & 12,7 & $10,6-14,6$ \\
\hline Falta de tiempo & 380 & 36,5 & $33,6-39,5$ \\
\hline Costumbre & 391 & 37,6 & $34,6-40,5$ \\
\hline Dieta & 46 & 4,4 & $3,2-5,7$ \\
\hline Disponibilidad & 148 & 14,4 & $12,1-16,4$ \\
\hline Problemas de salud & 21 & 2 & $1,2-2,9$ \\
\hline \multicolumn{4}{|l|}{ Motivo para elegir un alimento } \\
\hline Por gusto & 732 & 70,4 & $67,6-73,2$ \\
\hline Costos & 154 & 14,8 & $12,6-17,0$ \\
\hline Calidad & 113 & 10,9 & $9,0-12,8$ \\
\hline Valor Nutritivo & 147 & 14,1 & $12,0-16,3$ \\
\hline Recomendaciones médicas & 24 & 2,3 & $1,4-3,2$ \\
\hline Recomendaciones nutricionales & 51 & 4,9 & $3,6-6,2$ \\
\hline Otros motivos & 69 & 6,6 & $5,1-8,1$ \\
\hline \multicolumn{4}{|l|}{ Alteraciones de su salud percibidas } \\
\hline Gastritis & 313 & 30,1 & $27,3-32,8$ \\
\hline Dolor de Cabeza y Migraña & 285 & 27,4 & $24,7-30,1$ \\
\hline Problemas de Colon & 155 & 14,9 & $12,7-17,1$ \\
\hline Hipoglicemia, hiperglicemia, hipotiroid. & 6 & 0,6 & $0,1-1,0$ \\
\hline Dolores abdominales y de estomago & 148 & 14,2 & $12,1-16,4$ \\
\hline Anemia & 42 & 4 & $2,8-5,2$ \\
\hline Dolor en otras partes (espalda, cadera, hombros) & 95 & 9,1 & $7,4-10,9$ \\
\hline Ninguno & 98 & 9,4 & $8,9-9,2$ \\
\hline \multicolumn{4}{|l|}{ Conoce el consumo de sus calorías } \\
\hline Siempre & 25 & 2,4 & $1,5-3,3$ \\
\hline Casi siempre & 68 & 6,5 & $5,0-8,0$ \\
\hline Algunas veces & 220 & 21,2 & $18,7-23,6$ \\
\hline Casi nunca & 195 & 18,8 & $16,4-21,1$ \\
\hline Nunca & 532 & 51,2 & $48,1-54,2$ \\
\hline \multicolumn{4}{|l|}{ Consecuencias del estrés en sus hábitos } \\
\hline Ingiere más alimentos & 250 & 24 & $21,4-26,6$ \\
\hline Ingiere menos alimentos & 374 & 36 & $33,0-38,9$ \\
\hline Ingiere los mismos alimentos & 344 & 33,1 & $30,2-35,9$ \\
\hline No ingiere alimentos & 72 & 6,9 & $5,4-8,5$ \\
\hline \multicolumn{4}{|l|}{ Frecuencia de actividad física } \\
\hline $1-2$ veces por semana & 299 & 28,8 & $26,0-31,5$ \\
\hline $3-4$ veces por semana & 175 & 16,8 & $14,5-19,1$ \\
\hline 5 o más veces & 103 & 9,9 & $8,1-11,7$ \\
\hline No realiza & 463 & 44,5 & $41,5-47,5$ \\
\hline \multicolumn{4}{|l|}{ Peso al ingresar a la universidad } \\
\hline Se ha mantenido & 377 & 36,3 & $33,3-39,2$ \\
\hline Ha disminuido & 405 & 38,9 & $36,0-41,9$ \\
\hline Ha aumentado & 258 & 24,8 & $22,2-41,9$ \\
\hline \multicolumn{4}{|l|}{ Susceptible de Enfermar } \\
\hline $\mathrm{Si}$ & 255 & 23,1 & $20,0-26,0$ \\
\hline No & 785 & 76,9 & $74,0-79,9$ \\
\hline
\end{tabular}

Tabla 4. Frecuencia de factores relacionados con los hábitos alimenticios en los sujetos de estudio (Cartagena, 2011).

información obtenida. Esto fue soportado por la resolución de Helsinki, modificación de Edimburgo 2000 y resolución 8430, antiguo Ministerio de Salud, Colombia 1993.

Los datos fueron analizados con el programa estadístico STATA, versión para Windows 10.0. Se estimó la ocurrencia de los hábitos y trastornos alimenticios a través de prevalencias, asumiendo intervalos de confianza del 95\%. El análisis bivariable se realizó a través de razones de disparidad, utilizando los intervalos de confianza y la prueba chi-cuadrado para la significación estadística, con un límite de decisión de 0.05 . Por último, se realizó análisis multivariable a través de regresión logística nominal. Todos los estimadores fueron ajustados a partir del diseño muestral (afijación proporcional por programa). 


\begin{tabular}{|c|c|c|c|c|c|c|}
\hline & \multicolumn{2}{|c|}{ Riesgo de Anorexia/Bulimia } & \multicolumn{2}{|c|}{ Divariado } & \multicolumn{2}{|c|}{ Multivariable } \\
\hline & SI & NO & $\mathrm{OR}^{* *}$ & IC $95 \%$ & $\mathrm{OR}^{* * *}$ & IC $95 \%$ \\
\hline \multicolumn{7}{|l|}{ Factores influyentes } \\
\hline Edad menor de 19 años & 87 & 406 & $1,7^{*}$ & $1,192,42$ & $1,86^{*}$ & $1,26-2,77$ \\
\hline Sexo masculino & 94 & 433 & $1,84^{*}$ & $1,28-2,64$ & - & - \\
\hline Ingresos económicos de un SMLMV & 35 & 270 & 0,71 & $0,48-1,07$ & - & - \\
\hline Susceptibilidad de enfermar & 53 & 202 & $1,9^{*}$ & $1,31-2.76$ & $1,77^{*}$ & $1,17-2,70$ \\
\hline \multicolumn{7}{|l|}{ Motivo de sus hábitos alimenticios } \\
\hline Dificultades económicas & 25 & 107 & 1,5 & $0,93-2,39$ & - & - \\
\hline Falta de tiempo & 53 & 327 & 0,96 & $0,64-1,31$ & - & - \\
\hline Costumbre & 53 & 338 & 0,91 & $0,64-1,31$ & - & - \\
\hline Dieta & 14 & 32 & $2,8^{*}$ & $1,46-5,4$ & $2,81^{*}$ & $1,29-6,10$ \\
\hline Disponibilidad & 21 & 127 & 0,99 & $0,60-1,64$ & - & - \\
\hline Problemas de salud & 3 & 18 & 1.00 & $0.29-3,45$ & - & - \\
\hline \multicolumn{7}{|l|}{ Motivo para elegir los alimentos } \\
\hline Por gusto & 105 & 627 & 1,03 & $0,70-1,51$ & - & - \\
\hline Costos & 26 & 128 & 1,27 & $0,80-2,02$ & - & - \\
\hline Calidad & 17 & 96 & 1,07 & $0,62-1,86$ & - & - \\
\hline Valor nutritivo & 28 & 119 & 1,51 & $0,96-2,39$ & - & - \\
\hline Recomendaciones médicas & 3 & 21 & 0,85 & $0,25-2,91$ & - & - \\
\hline Recomendaciones nutricionales & 8 & 43 & 1,12 & $0,52-2,45$ & - & - \\
\hline \multicolumn{7}{|l|}{ Deterioro de la Salud } \\
\hline Gastritis & 48 & 265 & 1,13 & $0,78-1,65$ & - & - \\
\hline Dolor de Cabeza y Migraña & 46 & 239 & 1,23 & $0,84-1,80$ & - & - \\
\hline Problemas de Colon & 40 & 115 & $2,5^{\star}$ & $1,66-3,79$ & $1,81^{*}$ & $1,12-2,91$ \\
\hline Hipoglicemia, hiperglicemia, hipotiroid. & 3 & 3 & $6,13^{*}$ & $1,23-3,07$ & - & - \\
\hline Dolores abdominales y de estomago & 28 & 120 & 1,5 & $0,95-2,36$ & - & - \\
\hline Anemia & 113 & 29 & $2,86^{*}$ & $1,45-5,65$ & - & - \\
\hline Dolor en otras partes & 17 & 78 & 1,35 & $0,78-2,36$ & - & - \\
\hline \multicolumn{7}{|l|}{ Frecuencia de Actividad Física } \\
\hline No realiza actividad física & 99 & 364 & $2,93^{*}$ & $2,03-4,23$ & $3,04^{*}$ & $2,01-4,58$ \\
\hline
\end{tabular}

Tabla 5. Análisis de regresión entre trastornos de alimentación (SCOFF) y factores socio-demográficos, físicos y conductuales en los sujetos de estudio (Cartagena, 2011). * Significación estadística $(p<0,05)$; ** estimadores ajustados por diseño muestral (afijación proporcional por programas académicos); *** estimadores ajustados por regresión logística.

\section{RESULTADOS}

Los participantes en el presente estudio tenían un promedio de edad de 23 años (DE: 8,5). La distribución de las características de la muestra aparece en la tabla 1. Por otro lado, 22 estudiantes rechazaron su participación en el estudio, lo que corresponde al $2,1 \%$.

Con respecto a los hábitos alimenticios, cerca de la mitad de los participantes consumen tres comidas al día (44,9\%). También se observa una mayor frecuencia de estudiantes que consumen comidas en horas adecuadas (35,7\%), sin embargo, para el desayuno la mayoría lo hacen entre las 8 a.m. y las 9 a.m. (33,9\%). En este mismo sentido, solo el 4,5\% de los participantes manifestaron asistir al comedor universitario, programa que organiza la universidad de Cartagena con apoyo de la administración distrital. De estos, el 60,9\% consume el almuerzo en su lugar de residencia (tabla 2). De acuerdo al tipo de alimento consumido durante el día, para el desayuno se privilegian los azucares en $27,5 \%$.
Para el almuerzo y la cena, los alimentos cárnicos con $45,3 \%$ y $30,2 \%$ respectivamente (tabla 3 ). Para los alimentos consumidos entre comidas (media mañana o media tarde), con mayor frecuencia se consumen mecatos (pequeño refrigerio que se toma entre comidas), con $29,7 \%$. Además, el consumo de frutas solo se observa en el $13,2 \%$.

Entre los factores relacionados con los hábitos alimenticios, el motivo más frecuente para no alimentarse bien fue la falta de costumbre y el tiempo, con $37,6 \%$ y $36,5 \%$. Por otro lado, el principal motivo para elegir un alimento fue el gusto $(70,4$ $\%)$. Al mismo tiempo se evidenciaron deterioros en la salud relacionados con la alimentación, como gastritis $(30,1 \%)$, y ante situaciones estresantes el $36 \%$ de los estudiantes descuidan su nutrición e ingieren menos alimentos. La mayor parte de los participantes no realizan actividad física $(44,5 \%)$ (tabla 4).

La susceptibilidad de enfermar a través de la escala de Holmes And Rahe fue del 23,1\% (IC 95\%: 20,0- 


\begin{tabular}{|c|c|c|c|c|c|c|}
\hline & \multicolumn{2}{|c|}{ Riesgo de Anorexia/Bulimia } & \multicolumn{2}{|c|}{ Bivariado } & \multicolumn{2}{|c|}{ Multivariable } \\
\hline & SI & No & $\mathrm{OR}^{* *}$ & IC 95\% & $\mathrm{OR}^{* * *}$ & IC $95 \%$ \\
\hline \multicolumn{7}{|l|}{ Factores influyentes } \\
\hline Edad menor de 19 años & 40 & 53 & 1,15 & $0,73-1,81$ & - & - \\
\hline Sexo masculino & 52 & 475 & $1,97^{*}$ & $1,22-3,19$ & - & - \\
\hline Susceptibilidad de enfermar & 31 & 224 & $2,12^{*}$ & $1,32-3,42$ & $2,11^{*}$ & $1,27-3,51$ \\
\hline Ingresos económicos menos de un SMMLV & 32 & 273 & $1,71^{*}$ & $1,07-2,75$ & $2,33^{*}$ & $1,26-4,28$ \\
\hline \multicolumn{7}{|l|}{ Motivo de sus hábitos alimenticios } \\
\hline Dificultades económicas & 19 & 113 & $2,37^{*}$ & $1,37-4,13$ & - & - \\
\hline Falta de tiempo & 42 & 338 & $2,09^{*}$ & $1,32-3,32$ & $2,27^{*}$ & $1,37-3,77$ \\
\hline Costumbre & 17 & 374 & $0,43^{*}$ & $0,25-0,74$ & - & - \\
\hline Dieta & 10 & 36 & $3,72^{*}$ & $1,77-7,82$ & $6.0^{*}$ & $2,66-13,5$ \\
\hline Disponibilidad & 11 & 137 & 0,97 & $0,50-1,87$ & - & - \\
\hline Problemas de salud & 1 & 20 & 0,6 & $0,07-4,55$ & - & - \\
\hline \multicolumn{7}{|l|}{ Motivo para elegir los alimentos } \\
\hline Por gusto & 56 & 676 & 1,02 & $0,62-1,70$ & - & - \\
\hline Costos & 23 & 131 & $2,6^{*}$ & $1,55-4,37$ & - & - \\
\hline Calidad & 10 & 103 & 1,2 & $0,60-2,41$ & - & - \\
\hline Valor nutritivo & 11 & 136 & 0,98 & $0,51-1,90$ & - & - \\
\hline Recomendaciones médicas & 1 & 23 & 0,52 & $0,06-3,92$ & - & - \\
\hline Recomendaciones nutricionales & 3 & 48 & 0,75 & $0,23-2,47$ & - & - \\
\hline \multicolumn{7}{|l|}{ Deterioro de Salud } \\
\hline Gastritis & 25 & 288 & 1,08 & $0,66-1,77$ & - & - \\
\hline Dolor de Cabeza y Migraña & 18 & 267 & 0,76 & $0,45-1,32$ & - & - \\
\hline Problemas de Colon & 25 & 130 & $2,9^{*}$ & $1,78-4,92$ & $2,46^{*}$ & $1,4-4,33$ \\
\hline Hipoglicemia, hiperglicemia, hipotiroid. & 4 & 2 & $25,5^{*}$ & $4,61-41,9$ & - & - \\
\hline Dolores abdominales y de estomago & 15 & 133 & 1,45 & $0,81-2,64$ & - & - \\
\hline Anemia & 13 & 29 & $6,3^{*}$ & $3,14-12,7$ & $6,52^{*}$ & $2,94-14,5$ \\
\hline Dolor en otras partes & 8 & 87 & 1,13 & $0,53-2,43$ & - & - \\
\hline \multicolumn{7}{|l|}{ Frecuencia de Actividad Física } \\
\hline No realiza actividad física & 48 & 415 & $2,03^{*}$ & $1,27-3,26$ & $1,77^{*}$ & $1,06-2,94$ \\
\hline
\end{tabular}

Tabla 6. Análisis de regresión entre trastornos de alimentación (EAT-26) y factores socio-demográficos, físicos y conductuales en los sujetos de estudio (Cartagena, 2011). * Significación estadística $(p<0,05)$; ** estimadores ajustados por diseño muestral (afijación proporcional por programas académicos); ${ }^{\star * *}$ estimadores ajustados por regresión logística.

26,0). La frecuencia de individuos que presentaron riesgo de trastornos alimenticios, según el EAT26 , fue del $7,8 \%$ (IC 95\%: 5,9-9,8) y para SCOFF (anorexia y bulimia) del 14,2\% (IC 95\%: 11,7-16,7).

A nivel bivariable, al asociar el riesgo de trastornos de la alimentación según el SCOFF con variables socio-demográficas, físicas y conductuales, se encontró asociación estadística con la edad (OR: 1,7; IC 95\%: 1,19-2,42; $\mathrm{p}=0.003$ ), observándose mayor riesgo en menores de 19 años, y con el sexo (OR: 1,84; IC 95\%: 28-2,64; p=0.001), siendo mayor los valores en el sexo masculino. Además, también hubo significación estadística con susceptibilidad de enfermar (OR: 1,9; IC 95\%: 1,31-2,76; $p=0.001$ ), dieta (OR: 2,8; IC 95\%: 1,46-5,4; $\mathrm{p}=0.002$ ), problemas de colon (OR: 2,5; IC 95\%: 1,66-3,79; $p=0.01$ ), anemia (OR: 2,86; IC 95\%: 1,45-5,65; $\mathrm{p}=0.002$ ) y no realizar actividad física (OR: 2,93; IC 95\%: 2,03-4,23; $p=0.000$ ). Para las demás variables no se encontró significación estadística. En el análisis multivariable el mejor modelo mostró significación estadística $(p=0.000)$ al conjugar las siguientes variables: edad (OR: 1,86; IC 95\%: 1,262,$77 ; p=0.002$ ), susceptibilidad de enfermar (OR: 1,77; IC 95\%: 1,17-2,70; $p=0.000$ ), dieta (OR: 2,81; IC 95\%: 1,29-6,10; $p=0.009$ ), problemas de colon (OR: 1,8; IC 95\%: 1,12-2,91; $\mathrm{p}=0.01$ ) y no realizar actividad física (OR: 3,04; IC 95\%: 2,01-4,58; $\mathrm{p}=0.000$ ) (tabla 5).

Al asociar el riesgo de trastornos de la alimentación según el EAT-26 con variables socio-demográficas, físicas y conductuales, se encontró asociación estadística para el sexo (OR: 1,97; IC 95\%: 1,223,$19 ; p=0.006$ ), siendo mayor la probabilidad para los hombres, y con los ingresos económicos (OR: 1,71; IC 95\%: 1,26-4,28; $p=0.02$ ), observándose mayor probabilidad de trastornos en los estudiantes cuyos padres tienen ingresos menores a un salario mínimo mensual legal vigente (SMMLV). Por otro lado, hubo significación con la susceptibilidad de enfermar (OR: 2,12; IC 95\%: 1,27-3,51; $p=0.002$ ), dificultades económicas (OR: 2,37; IC 95\%: 1,374,13; $p=0.002)$, falta de tiempo para alimentarse (OR: 2,09; IC 95\%: 1,32-3,32; $p=0.002$ ), falta 
de costumbre (OR: 0,43; IC 95\%: 0,25-0,74; $\mathrm{p}=0.003$ ), dieta (OR: 3,72; IC 95\%: 1,77-7,82; $p=0.001)$, elección de alimentos por costos (OR: 2,6; IC 95\%: 1,55-4,37; $p=0.000$ ), problemas de colon (OR: 2,9; IC 95\%: 1,78-4,92; $\mathrm{p}=0.000$ ), hipoglucemia e hipotiroidismo (OR: 25,5; IC 95\%: 4,61-41,9; $p=0.000$ ) y anemia (OR: 6,3; IC 95\%: 6,14-12,7; $p=0.000)$. Además, con no realizar actividad física (OR: 2,03; IC 95\%: 1,273,$06 ; p=0.003$ ). Para las demás variables no se encontró significación estadística. En el análisis multivariable, el mejor modelo mostró significación estadística $(p=0.000)$ al conjugar las siguientes variables: ingresos económicos (OR: 2,33; IC 95\%: 1,26-4,28; $p=0.007$ ), susceptibilidad de enfermar (OR: 2,11; IC 95\%: 1,27-3,51; $p=0.004$ ), falta de tiempo para alimentarse (OR: 2,27; IC 95\%: 1,373,77; $p=0.001$ ), dieta (OR: 6,0; IC 95\%: 2,66-13,5; $p=0.000$ ) problemas de colon (OR: 2,46; IC 95\%: 1,4-4,32; $p=0.002$ ), anemia (OR: 6,52; IC 95\%: $2,94-14,5 ; p=0.000)$ y no realizar actividad física (OR: 1,77; IC 95\%: 1,06-2,94; p=0.002) (tabla 6).

\section{DISCUSIÓN}

Los desórdenes alimenticios han sido ampliamente estudiados en escolares y estudiantes de secundaria, pero muy poco en estudiantes universitarios. De ahí la importancia de conocer este comportamiento para implementar acciones que mejoren los estilos de vida en adultos jóvenes, población altamente productiva en todos los países.

Con respecto a la validez interna del presente estudio, para evitar un sesgo de selección se realizó un muestreo con afijación proporcional por programas y semestres. Por otro lado, debido a las características de los instrumentos autoadministrados, es posible que exista sesgo de memoria, el cual se intentó prevenir explicando a los participantes la intención de cada pregunta expresada en el formulario. Así mismo, los formatos de encuestas fueron calibrados en un pilotaje con un grupo de estudiantes que no formaban parte de la población de estudio, pero con características similares. Acada ítem se le valoró el grado de validez y consistencia, para posteriormente aplicarlos en los individuos seleccionados. Además, a los cuestionarios construidos por los investigadores se les evaluó su validez de apariencia, a través de una valoración por tres jueces expertos, teniendo en cuenta su pertinencia, suficiencia de las categorías, plausibilidad, semántica, sintaxis y ordenamiento de los ítems, permitiendo adecuarlo al lenguaje de la ciudad de Cartagena para mejorar su comprensión e interpretación.
En el presente estudio se encontró que los jóvenes consumen siempre sus tres comidas y acostumbran a desayunar, pero éste se consume a media mañana, cuando ya ha avanzado la jornada académica, siendo lo cotidiano desayunar antes de iniciar la jornada diaria (horario de las 7 a.m.). En este sentido, la gran mayoría de los estudiantes consumen su primera comida entre las 8 y 9 a.m. Estos hallazgos son similares a los reportados por Ibáñez ${ }^{10}$ en estudiantes de odontología de la Fundación Universitaria San Martín de Bogotá, Colombia, donde el 67,6 \% de los participantes no desayunan antes de iniciar sus labores académicas. De esta forma se percibe la irregularidad en la alimentación en estas poblaciones, cuyos sujetos de estudio no siguen un régimen alimenticio regular y consumen la mayoría de productos durante la segunda mitad del día. Por otro lado, el $78,8 \%$ de los estudiantes consumen más de cinco comidas principales diarias, teniendo en cuenta que en países caribeños, como Colombia, se consumen tres comidas principales y dos comidas pequeñas a media mañana y media tarde. Al comparar con los resultados obtenidos en estudiantes argentinos ${ }^{11}$, éstos consumen cuatro comidas diarias en un $37,8 \%$, con horarios irregulares en un $49,8 \%$. Además, en estudiantes chinos $^{12}$ el consumo de tres principales comidas al día fue el más frecuente (79\%). En estudiantes colombianos, en la ciudad de Cali ${ }^{13}$, se reportó que el $42,7 \%$ de los individuos consumen las tres principales comidas diarias.

Durante el almuerzo, el 60,9 \% de los estudiantes encuestados en el presente estudio prefieren comer en su lugar de residencia, y además más de la mitad de ellos consumen la cantidad de agua recomendada para el buen funcionamiento del organismo. No obstante, el consumo de frutas es bajo y se observa un alto consumo de comidas rápidas (embutidos, fritos, hamburguesas) y mecatos (pequeños refrigerios que se toman entre comidas). Estos resultados son contrarios a los reportados por Troncoso et $\mathrm{a}^{1 / 4}$ en estudiantes universitarios, quienes encontraron consumo de jugos de frutas en el $47,6 \%$ y consumo esporádico de comidas rápidas y papas fritas.

En el presente estudio, para el desayuno se privilegian los azúcares y para el almuerzo y la cena los alimentos cárnicos. En un estudio realizado en Barcelona, España ${ }^{15}$, se encontraron consumos superiores al $50 \%$ de azúcares y cárnicos, así como refrescos con y sin gas. Cabe resaltar que estos estudiantes también manifestaron consumo 
de frutas y verduras en niveles insuficientes, coincidiendo también con los resultados de la presente investigación.

Por otro lado, en el presente estudio, el 70,4\% de estos estudiantes seleccionan sus alimentos por gusto propio y no por calidad o valor nutritivo, a diferencia de los hallazgos encontrados en estudiantes de la Universidad Juárez Autónoma de Tabasco, Mèxico ${ }^{16}$, quienes en la determinación de la elección de alimentos encuentran los factores económicos. En este sentido, este grupo de estudiantes destinaban para la alimentación \$20,00 pesos diarios mexicanos, lo que hace pensar que quizás muchos de los que asisten a universidades públicas no cuentan con los recursos suficientes para satisfacer sus necesidades alimentarias y nutritivas. En este sentido, en el ambiente universitario se pueden encontrar muchas opciones no saludables, pero es sólo decisión de las personas saber seleccionar inteligentemente su alimentación, cambiando su estilo de vida.

Muchos de los universitarios del presente estudio consideran que no presentan una alimentación saludable y el $32,6 \%$ de ellos perciben que presentan hábitos alimenticios deficientes para sus necesidades, que obedecen a la falta de tiempo en un $36,5 \%$ y a la costumbre en un $37,6 \%$. Ésta podría ser la justificación a la disminución de peso en el $38,9 \%$ de los participantes, con respecto al valor obtenido antes de ingresar a la Universidad. En jóvenes universitarios pertenecientes a la Universidad Católica de la Santísima Concepción, ubicada en la región del Bío-Bío, Chile ${ }^{17}$, también se manifiestan similares motivos para no presentar una alimentación saludable.

Al evaluar otros estilos de vida, además de no encontrarse una alimentación saludable para la edad, en los sujetos de este estudio se encontró que el $44,5 \%$ no realizan actividad física, lo que puede en el futuro fomentar la aparición de sobrepeso y obesidad. Esto coincide con lo reportado en estudiantes encuestados de la Pontificia Universidad Católica de Valparaíso, Chile $^{18}$, quienes en un $53 \%$ no realizan ejercicio. Además, los universitarios chilenos presentaron descuido en su alimentación por situaciones de estrés, al igual que en el presente estudio, en cuyos estudiantes hubo un alta susceptibilidad de enfermar (escala de Holmes and Rahe $)^{9}$. Esto explica que los participantes ante situaciones estresantes familiares descuiden su nutrición, ingiriendo menos alimentos y de baja calidad.
Los deterioros de la salud, como gastritis, dolor de cabeza y migraña, problemas de colon y dolores abdominales, se presentaron con una frecuencia importante en el presente estudio. En este sentido también se coincide con Ibáñez et $a / 5$, quienes reportaron estas alteraciones, aunque con algunas diferencias entre sus proporciones (gastritis 55.3\%, dolor de cabeza y migraña $13.2 \%$ y problemas de colon $9.2 \%$ ).

Con respecto a la prevalencia de desórdenes alimenticios presentados posteriormente a la aplicación del test de actitudes alimentarias (SCOFF), se encontró anorexia o bulimia en el $14,2 \%$. Este dato es inferior al obtenido por Ruedas et $a{ }^{18}$ en la Universidad Autónoma de Bucaramanga, Colombia, donde el 38,7 \% presentó puntuaciones positivas. En este sentido, el cuestionario SCOFF parece ser una buena alternativa como instrumento para detectar los trastornos de la conducta alimentaria en universitarios. Cabe resaltar que estas pruebas son de despistaje y se hace necesaria la confirmación de estos riesgos a través de un diagnóstico psiquiátrico. En el presente estudio se utilizó el cuestionario SCOFF, después de hacer una exhaustiva revisión de la literatura. Estudios de Morgan $^{8}$ y Ruedas ${ }^{18}$ realizados en universitarios han descrito la consistencia interna y la validez de criterio de este cuestionario, concluyendo que la prueba presenta sensibilidad por encima de $90 \%$ y especificidad por encima de $80 \%$, siendo una buena alternativa para el despistaje de los trastornos alimenticios en universitarios. Estos resultados son contrarios a los obtenidos por Cogollo et al ${ }^{19}$, quienes concluyen que la consistencia interna del cuestionario SCOFF es notablemente baja en adolescentes estudiantes de un colegio de bachillerato de la ciudad de Cartagena. Por ser los participantes en el presente estudio jóvenes universitarios, se tomaron como referencia otros resultados nacionales en poblaciones de universitarios ${ }^{8,18}$.

Por otro lado, al ser el EAT-26 una prueba de despistaje, en el presente estudio no se utilizó como un instrumento definitivo en la identificación de trastornos de la conducta alimentaria. Sin embargo, algunos estudios han demostrado que esta prueba puede ser una eficiente herramienta de monitorización como parte de un proceso de screening, teniendo en cuenta dos fases. A pesar de esto, el EAT-26 es una prueba con valor predictivo positivo bajo, por lo que se requiere utilizar otros instrumentos complementarios para detectar conductas características de los tras- 
tornos alimenticios y evitar subregistros. Por ello, se consideró aplicar primero el test EAT-26 y luego la prueba de SCOFF. Esta última ofrece un despistaje más seguro y por ende, al usarse ambas en los análisis bivariados, coincidieron en las significaciones estadísticas de los estimadores obtenidos para algunos factores considerados de riesgo para los trastornos alimenticios.

En el presente estudio se encontró significación estadística al relacionar estos puntajes positivos del SCOFF con la edad, existiendo mayor probabilidad de trastornos en los menores de 19 años (OR: 1.7), contrario a lo reportado por Manrique et $a^{20}$, quienes encontraron un OR: 0.69. Esto supone la necesidad de considerar al adolescente como vulnerable y en riesgo de padecer muchas adicciones y trastornos, por las condiciones psicosociales propias de esta etapa del ciclo vital individual, lo que permite realizar un seguimiento más de cerca en todas sus dimensiones. Según Kaunas et $a^{21}$, la tarea principal de la adolescencia es lograr la identidad personal, entendiendo ésta como un proceso de constante construcción. Según este proceso, "el individuo se juzga a sí mismo a la luz de lo que percibe como la manera en que los demás lo juzgan a él comparándolo con ellos".

Por último, se observaron relaciones estadísticamente significativas entre el riesgo de padecer anorexia y bulimia según el SCOFF con la susceptibilidad de enfermar. Además, hubo significación estadística en la relación con problemas de colon, anemias y dietas y con el hecho de no realizar actividad física. Estos hallazgos son similares a los reportados por Manrique et $a l^{19}$, quienes observaron que todas las actuales prácticas dietéticas en la población estudiantil (OR: 8.76) son la causa generadora de estos problemas de salud. Se hace conveniente entonces remitir a esta población a un diagnóstico psiquiátrico para confirmar los resultados por los problemas adicionales que se están presentando y que pueden ser el origen o la consecuencia de padecer anorexia y bulimia ${ }^{22-24}$.

Los resultados del presente trabajo pueden servir de guía para consolidar políticas integrales de bienestar estudiantil en los universitarios, donde se puedan explorar los factores que influyen en este problema y acceder a ayudas psicológicas que permitan un manejo profesional de estas exposiciones dentro de cada periodo académico.

A partir de los hallazgos observados, se concluye que existe presencia de trastornos de alimentación y hábitos inadecuados en estudiantes universitarios y que éstos se relacionan con algunos factores académicos y conductuales típicos del quehacer universitario, siendo necesario considerar este comportamiento como un problema que produce complicaciones físicas y psicosociales y que puede llevar a la incapacidad para continuar el desarrollo de actividades académicas.

\section{AGRADECIMIENTOS}

A las directivas y estudiantes de la Universidad de Cartagena por su contribución en la realización de este trabajo, el cual fue conducido por el Grupo de Salud Pública GISPOUC y permitió la formación de un estudiante en la Maestría de Salud Pública de la Universidad Nacional de Colombia.

\section{BIBLIOGRAFÍA}

1. Álvarez Rosario J, Castaño Castrillón J, Marín Viatela J, Navas Galvis C, Noreña Vidal P, Ovalle Arciniegas H, et al. Estilos de vida en estudiantes de la universidad de Manizales 2006. Arch Med Manizales. 2007; 7:46-56.

2. Marín V. Trastornos de la conducta alimentaria en escolares y adolescentes. Rev Chil de Nutri. 2002; 2:86-91.

3. Hoek H, Van Hoeken D. Review of the prevalence and incidence of eating disorders. Inter $\mathrm{J}$ of Eating Disorders. 2003; 34:383-96

4. Steinhausen $\mathrm{H}$, Boyadjieva S, Grigoroiu-Serbanescu M, Seidel R, Winkler Metzke C. A transcultural outcome study of adolescent eating disorders. Acta Psychiatrica Scand. 2002; 101:60-6.

5. Sanabria P, González L, Urrego D. Estilos de vida saludable en profesionales de la salud colombianos estudio exploratorio. Revista Médica. 2007; 15:207-17.

6. Ángel LA, Vásquez R, Chavarro K, Martínez LM, García J. Prevalencia de trastornos del comportamiento alimentario en estudiantes de la Universidad Nacional de Colombia. Acta Med Colomb. 1997; 22:111-9.

7. Garner DM, Olmsted MP, Bohr, YY Garfinkel PE. The Eating Attitudes Test: Psychometric features and clinical correlates. Psychological Medicine. 1982; 12:871-8.

8. Morgan JF, Reid F, Lacey JH. Screeninf tool for eatíng disorders. The SCOFF questionnaire: assessment of a new. BMJ 1999; 31:1467-8.

9. Brunner C, Acuña L, Gallardo L, Atri R, Hernandez A. La escala de Reajuste Social de Holmes and Rahe en México. Rev Latinoamericana de Psicologia. 1994; 26:253-69.

10. Ibáñez E, Thomas $Y$, Bicenty A, Barrera J. Cambios de hábitos alimentarios de los estudiantes de odontología de la Fundación Universitaria San Martín de Bogotá. Ciencias Biomédicas. 2008; 6:101-212.

11. Horacio Gerometta P, Carrara C, Alberto Galarza L, Feyling V. Frecuencia de consumo de alimentos en ingresantes a la carrera de medicina: Rev posgrado de la VI cátedra de medicina. 2004;136:9-13.

12. Sakamaki R, Toyama K, Amamoto R, Liu Cj, Shinfuku N. Nutritional knowledge, food habits and health attitude of Chinese university students a cross sectional study. Nutr J. $2005 ; 4: 4$. 
13. Tobar Vargas L, Vásquez Cardoso S, Bautistas Muñoz L. Descripción de hábitos y comportamientos alimentarios de los estudiantes de la Facultad de Ciencias de la Pontificia Universidad Javeriana, 2005. Universitas Scientiarum. 2008; 13:55-63.

14. Troncoso PC, Amaya JP. Factores sociales en las conductas alimentarias de estudiantes universitarios. Rev Chil Nutr. 2006; 36:1090-7.

15. Díaz M M, Montserrat RA, Gálvez M. Patrón alimentario de estudiantes universitarios: Comparación entre culturas. Rev Esp Nutr Comunitaria. 2005; 11:8-11.

16. Barragán M. Hábitos alimentarios en estudiantes de la Universidad Juárez Autónoma de Tabasco. Rev Cubana Salud Pública. 2006; 32:656.

17. Macmillan KN. Valoración de hábitos de alimentación, actividad física y condición nutricional en estudiantes de la pontificia universidad católica de Valparaíso. Rev Chil Nutr. 2007; 34:56

18. Rueda GE, Díaz LA, Ortiz D, Pinzón C, Rodríguez J, Cadena LP. Validación del cuestionario SCOFF para cribado de trastornos del comportamiento alimentario en adolescentes escolarizadas. Aten Primaria. 2005; 35:89-94.

19. Campos Arias A, Cogollo Z, Díaz C. Cuestionario SCOFF para tamizaje de trastornos de la conducta alimentaria, Consistencia interna en estudiantes de la ciudad de Cartagena. Revista de Facultad de Ciencias de la Salud. Rev DUAZARY. 2007; 4:14-8.

20. Manrique G, Barrera L, Ospina J. Prevalencia de Bulimia y Anorexia en adolescentes de Tunja y algunos factores de riesgo probablemente asociados. Avances en Enfermería. 2006; 24:38-46.

21. Skemiene L, Ustinaviciene R, Piesine L, Radisauskas R. Peculiarities of medical students' nutrition. Medicina (Kaunas) 2007; 43:145-52.

22. Kreipe RE, Birndorf SA. Eating disorders in adolescents and young adults. Med Clin North Am. 2000; 84:1027-49.

23. Becker AE, Grinspoon SK, Klibanski A, Herzog DB. Eating disorders. N Engl J Med. 1999; 340:1092-8.

24. Uramowska-Zyto B, Kozłowska-Wojciechowska M, Jarosz A, Makarewicz-Wujec M. Dietary and life-style habits of university students in Poland - empirical study. Rocz Panstw Zakl Hig. 2004; 55:171-9. 